\title{
TURISMO, PATRIMONIO TERRITORIAL Y DESARROLLO EN EL MEDIO ATLAS NORORIENTAL (MARRUECOS $)^{1}$
}

\author{
Enrique López Lara* \\ Universidad de Sevilla \\ Abdellatif Tribak** \\ Hanane Baali** \\ Lamiae El Bezzari*** \\ Université Sidi Mohamed Ben Abdellah. Marruecos
}

\section{RESUMEN}

Las montañas del nordeste del Medio Atlas marroquí constituyen una zona frágil y aislada, donde la población presenta precarias condiciones de vida. Desde el punto de vista natural y cultural, ocupan un lugar relevante en el conjunto de las montañas marroquíes al contar con recursos territoriales y patrimoniales. El turismo constituye una alternativa viable para activar el desarrollo económico y social. En este artículo se plantean las luces y las sombras que tendría la puesta en marcha de actividades propias del turismo, con un enfoque participativo y descentralizado.

Palabras clave: turismo, patrimonio territorial, desarrollo, Medio Atlas Nororiental.

Recibido: 2 de mayo de 2016

Devuelto para su revisión: 4 de octubre de 2016

Aceptado: 20 de noviembre de 2016

* Facultad de Geografía e Historia. . Universidad de Sevilla. C/ Doña María de Padilla, s/nº 41001 SEVILLA (España).E-mail:elopezl@us.es

** Faculté des Lettres et des Sciences Humaines. LAGEA-DD. Université Sidi Mohamed Ben Abdellah. Route d'Imouzzer. BP 59 Fes-Saïs.30000. Marruecos. E-mail: tribakabdellatif@yahoo.fr ; baalihanane@hotmail. fr ; lam.elbezzari@gmail.com

1 El artículo es producto del Proyecto conjunto de Investigación A/023361/10 "Ecoturismo y el patrimonio paisajístico y cultural como recurso para el desarrollo territorial y local del Medio Atlas y del Rif”, entre las Universidades de Sevilla y Sidi Mohamed Ben Abdellah de Fez, patrocinado por la Agencia Española de Cooperación Internacional al desarrollo (AECId). 


\title{
Tourism, heritage territorial and development in the Northeastern Middle Atlas (Morocco)
}

\begin{abstract}
The mountains of northeastern Middle Atlas (Morocco) constitute a fragile and isolated area, where the population has precarious living conditions. From the point of natural and cultural, figure prominently in all the Moroccans to have territorial heritage resources and mountains. Tourism is a viable option to activate the economic and social development alternative. This article describes the lights and shadows that would start up of own tourism activities, with a participatory and decentralized approach arise.
\end{abstract}

Keywords: tourism, territorial heritage, development, Middle Atlas North East.

\section{INTRODUCCIÓN}

Marruecos detenta en sus variadas regiones montañosas (Rif, Medio Atlas, Alto Atlas, Anti-Atlas) una gran parte de sus recursos naturales y patrimoniales. Actualmente, estos espacios montañosos están en crisis y presentan condiciones de vida difíciles para sus pobladores, cuestión que se contrapone con la riqueza natural y ambiental y el valor patrimonial de los diferentes conjuntos regionales donde se ubican.

El nordeste del Medio Atlas se presenta hoy en día como un entorno territorial periférico y aislado dentro de Marruecos (Mapa 1), de difícil acceso, tradicionalmente descuidado en el devenir histórico por quienes lo han administrado, siempre desde fuera del propio territorio. Estos dos importantes hándicaps se combinan con el mismo entorno físico-geográfico y la desconfianza de la población local en función de su exclusión de las estrategias de desarrollo implantadas en Marruecos (López Lara y Otros, 2007a).

La exclusión de esta área del proceso nacional de desarrollo social y económico ha acentuado el desequilibrio de sus estructuras, al socavar sus recursos naturales y condenar a su población a quedar marginada dentro del movimiento estatal de desarrollo humano (Reino de Marruecos, 2005).

El nordeste del Medio Atlas posee fortalezas específicas que le dan una identidad propia y lo convierten en un territorio con importantes valores patrimoniales e intereses estratégicos. Cuenta con unos recursos naturales, humanos y culturales que constituyen la base de sus potencialidades: clima fresco, abundante nieve, valles de gran belleza paisajística y el carácter natural de ciertos lugares con rica diversidad florística y con formaciones kársticas, así como la pervivencia de prácticas locales y tradiciones culturales consuetudinarias (López y Tribak, 2013).

Cuestiones que pueden llegar a constituirse como atractivos turísticos de potenciales visitantes, tanto nacionales como internacionales. El territorio considerado también tiene otras actividades adicionales, principalmente relacionadas con el patrimonio y que pueden funcionar como activos para el desarrollo desde la perspectiva del ocio y del turismo (Silva y Fernández, 2010), como el agro-pastoreo, la artesanía, la gastronomía, las actividades culturales, etc. 


\section{Mapa 1 \\ LOCALIZACIÓN ZONA DE ESTUDIO: NORDESTE DEL MEDIO ATLAS}

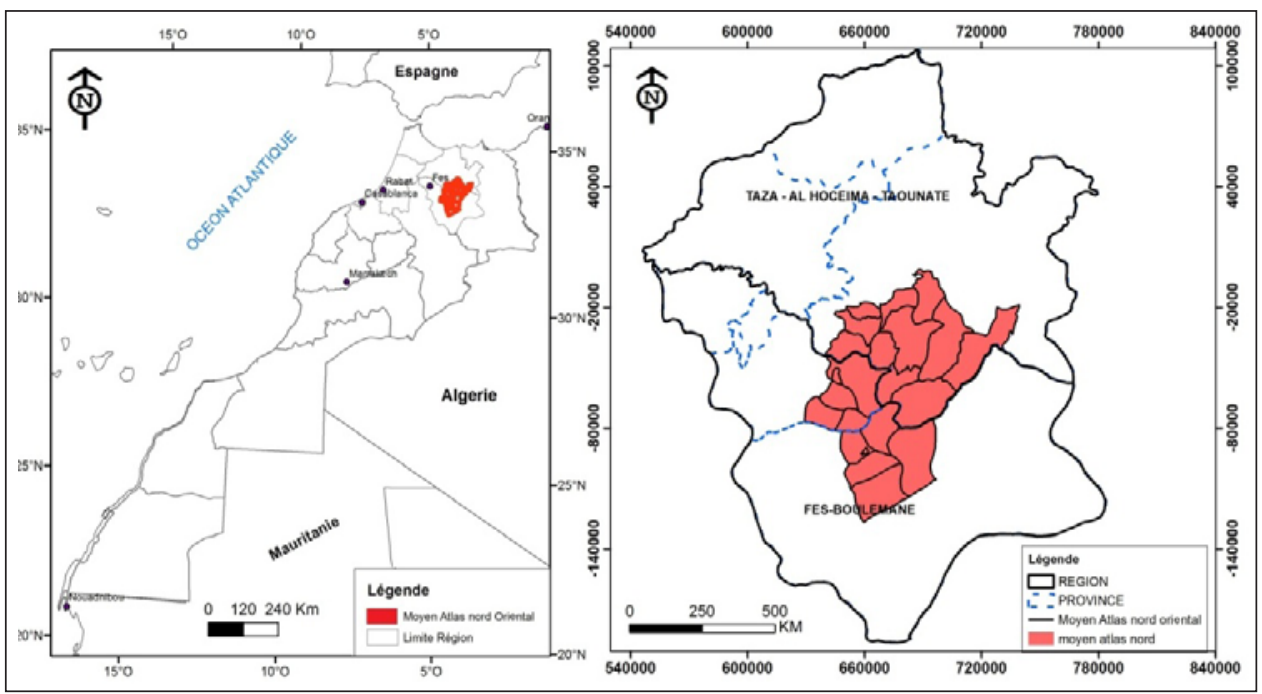

Fuente: elaboración propia.

Por esta razón, son lógicas las esperanzas que concitan las actividades turísticas para reactivar la economía e integrarla dentro del rango de las áreas de crecimiento favorecidas del país. Sin embargo, surgen preguntas relacionadas con las condiciones necesarias para el establecimiento de un desarrollo turístico que llegue a impulsar el crecimiento y desarrollo de estas zonas desfavorecidas (Cánoves y Otros, 2005) sin alterar sus valores patrimoniales y culturales.

Esta investigación se basa en su planteamiento y desarrollo en los siguientes cinco trabajos, diacrónicamente expuestos: 1) la hipótesis que establece que el turismo y los recursos territoriales pueden ser la base del desarrollo en los macizos orientales del Medio Atlas marroquí (Peyron, 2004); 2) los nuevos usos y visiones del espacio del turismo rural (Cánoves y Otros, 2005); 3) el dilema expuesto por Milian (2007) entre desarrollo y protección en las montañas del Medio Atlas; 4) el estudio comparado de casos diferentes en relación al patrimonio y al desarrollo sostenible real (Feria, 2010); y 5) los planteamientos teóricos y la metodología utilizadas por Troitiño y Troitiño (2016).

Se presenta en este trabajo los resultados finales de los proyectos de cooperación e investigación interuniversitaria Marruecos-España financiado por la AECId que ya dieron lugar a otros trabajos específicos (Tribak y Otros, 2010; López Lara y Tribak, 2013) donde se analizaban las oportunidades del Medio Atlas Oriental mediante, respectivamente, la realización de una guía turística y la creación de un itinerario pormenorizado para su visita y disfrute. Como colofón de la investigación y resultado final ha tenido lugar la defensa de dos tesis doctorales Baali (2014) y El Bezzari (2014). 


\section{UN PATRIMONIO TERRITORIAL SUSCEPTIBLE DE SER CONSERVADO}

El nordeste del Medio Atlas, ubicado en la parte meridional de la provincia de Taza, constituye una entidad geográfica diferenciada dentro la cadena del Atlas. Esta región, de altas montañas, arbolada en su mayor parte y majestuosa en su tamaño, presenta una originalidad paisajística y natural notable.

La zona es geológicamente, en su mayor parte, de estilo Jurásico, formándose cadenas de montañas plegadas, caracterizadas por la sucesión de amplios valles sinclinales (Tazarine, Tafferte, Tamtrouchte, Maghraoua, Gueldamane-Bechyine, Tamjout...) y anticlinales (Bou Iblane, Ramuz Rkibat, Chaara, Azrou Ouasser...), que generan alineaciones subparalelas, y hoy en día, representan los picos más altos de la cadena.

La zona presenta un dominio bioclimático de montaña mediterránea: colinas semiáridas en el oeste que contrastan con el norte -de subhúmedo a húmedo- de las altas cumbres de la cadena, con inviernos muy fríos y húmedos, en especial en los picos de Bou Iblane.

Al noreste y al este, empieza climatológicamente una transición de semiárido a árido. En invierno, la nieve es abundante, quedando constante y regular durante varias semanas en altitudes superiores a los $1200 \mathrm{~m}$ y, a veces, persiste hasta junio en los picos de Bou Iblane (Foto 1). La cubierta forestal es bastante abundante, con presencia de robles, alcornoques y cedros, que cubren grandes extensiones de las referidas montañas.

La población que la habita, en su mayoría de origen amazigh, presenta baja densidad (aproximadamente sobre los $40 \mathrm{hab} / \mathrm{km}^{2}$ ) con variaciones significativas entre las localidades según su ubicación a ladera o más arriba de las montañas. El crecimiento demográfico de la población es generalmente bajo e, incluso, con tendencia negativa en algunas localidades, por mor de la emigración.

El contexto sociocultural de la población del noreste del Medio Atlas junto al residuo de la estratificación histórico-cultural que homogeneiza las diferentes fases del desarrollo de la agricultura y su asentamiento en cuencas interiores (Tankraramt, Tamjilt, Tmourgout, Talzamt...) por las sociedades humanas sucesivas en la región a lo largo de la historia son elementos que conforman un carácter diferenciado y particular a la región del noreste del Medio Atlas .

El nordeste del Medio Atlas ofrece un interesante patrimonio territorial que puede llegar a representar un yacimiento para el desarrollo de las diferentes tipologías del turismo sostenible, ecológico, rural... (Silva y Fernández, 2008). La variedad del patrimonio territorial, así como la complementariedad natural y cultural, puede constituir ventajas absolutas, tanto materiales como inmateriales.

Los paisajes forestales son diversos y excelentes. Los bosques de cedros están aún bien conservados en torno a las áreas del lago Tamda y de Bou Iblane-Tafferte. Estos bosques ofrecen importantes oportunidades para el desarrollo agrícola y para las actividades del turismo ecológico. Además, proporcionan espacios para proyectos de oportunidades creativas, tales como parques regionales y nacionales de gran valor patrimonial y socioeconómico.

Los recursos de agua, aunque distribuidos de forma diferenciadas en función del contexto geológico y geomorfológico (López Lara y Otros, 2007b), en general abastecen bien a la región, dado el enorme potencial de reservas de macizos calcáreos del Atlas y el alcance de sus cuencas hidrográficas bien alimentadas por lluvias y nevadas. 


\section{Foto 1 MACIZO DE BOU IBLANE}

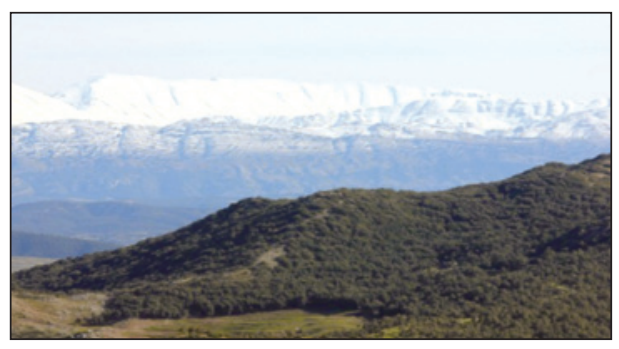

Foto 2 POLJÉ DAYT CHIKER

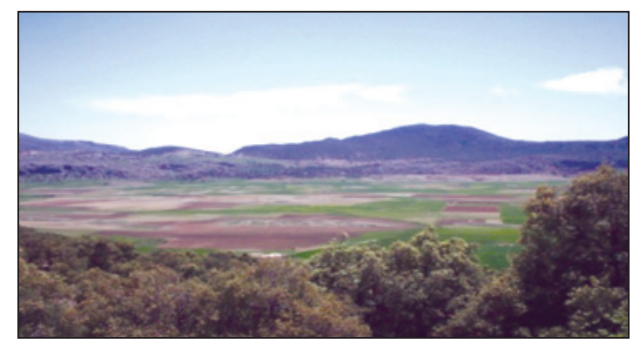

Los paisajes kársticos se encuentran por todas partes en las formaciones calcáreas y son un patrimonio natural innegable, otorgando a la región una identidad notable. La famosa cueva de Friouato y el Polje de Dayt Chiker son ejemplos sobresalientes (Foto 2).

Los enclaves reserva de Bou Iblane y Oued el Bared (Tametrochte; Tighza) son también notables S.I.B.E. (Sitios de Interés Biológicos Especiales) en la región. La primera, se trata de una alta montaña a $3172 \mathrm{~m}$, que agrupa riqueza florística y faunística. El ecosistema de cedro, bastante bien conservado, ofrece un gran potencial para el ecoturismo. La segunda, que es la fuente de Oued El Bared (Ain Ighz), está situada a unos $15 \mathrm{~km}$ al norte de Tankrarmt, formando unas poderosas y permanentes fuentes que brotan de una cueva profunda. Estas fuentes alimentan Oued El Bared y forman espectaculares gargantas.

El patrimonio construido, bastante variado y diverso, es un aspecto notable de la identidad de la región, que queda reflejada en la técnica arquitectónica que remonta su origen a varios siglos. Este tipo de hábitat refleja la adaptación de la población a las duras condiciones naturales del entorno. Se distingue por el uso de materiales locales y se basa en una arquitectura bioclimática capaz de ser fuente de frescor en los calurosos días de verano y fuente de calor en los duros días de invierno. Las estructuras hidráulicas ancestrales (acequias, pozos, colectores) siguen siendo funcionales en algunos aduares (Tribak y Otros, 2006).

Las actividades de artesanía y artísticas así como las tradiciones locales tienen el reconocimiento y representan una riqueza inminente, un componente esencial para el impulso del turismo en la región. El patrimonio cultural (folklore y costumbres) es rico y diverso, reflejando la fuerte relación entre el hombre y su medio ambiente.

Esta herencia juega un papel importante en la valorización de la cultura amazigh allí existente y un punto fuerte para la promoción del turismo rural de este territorio de montaña. El saber hacer local y los productos de artesanía diversifican los productos turísticos locales (Tribak, 2011).

La región es la sede de uno de los más antiguos Parques Naturales de Marruecos, el Parque Nacional Tazekka, que actualmente ocupa una superficie de 13.737 has, y ofrece una amplia variedad de paisajes geomorfológicos, flora y fauna. Los senderos paisajísticos son numerosos y atractivos: más de nueve rutas a pie se pueden realizar dentro del Parque de Tazekka (Bouslama, Bouhadli...). La diversidad de los paisajes de la región 
permite considerar otros senderos o rutas para descubrir un patrimonio natural y cultural, por ejemplo, en las comunas de Meghraoua, Zrarda, de Tazarine y de Bou Iblane, que ofrecen variadas posibilidades.

Se trata, en definitiva, de una región con valores naturales susceptibles de protección y conservación, sin entrar en conflicto, antes al contrario, con sus capacidades de desarrollo (Milian, 2007)

\section{REPERCUSIONES DE LA CRISIS: RUPTURA DE EQUILIBRIOS TRADI- CIONALES Y DETERIORO DEL PATRIMONIO TERRITORIAL}

Debido a su situación geográfica, sus caracteres geológicos, la orientación de sus laderas, de una parte, y de otra, la tradición socio-económica y cultural de sus pobladores, el nordeste del Atlas Medio presenta una variedad de recursos naturales que han asegurado durante mucho tiempo un equilibrio entre la dinámica socio-económica y ecológica locales.

Un ejemplo representativo se encuentra en el hábitat rural de montaña tradicional, producto de su relación con el medio natural que le da soporte y quien condiciona la organización, las formas arquitectónicas y los materiales utilizados, frecuentemente autóctonos, al extraerse del propio entorno geográfico natural, como en la propia construcción (López y Obda, 2012). Lo expuesto se sustenta en una dinámica regida por modos locales de producción basados en el conocimiento tradicional, que se ha mostrado capaz de combinar la organización económica, social y ecológica con el mantenimiento de un equilibrio armónico en la relación hombre-medio. Sin embargo, desde hace aproximadamente tres decenios, como consecuencia de la crisis que experimentan y viven los espacios de montaña de Marruecos, el desequilibrio del sistema económico y territorial local está haciendo clara mella en los caracteres ambientales. Ha de tenerse presente que la administración marroquí concentra la mayor parte de los esfuerzos, inversiones y actuaciones en el desarrollo de áreas rurales irrigadas, con la construcción de grandes presas. De la misma forma, por mor del éxodo rural, dedica esfuerzos al desarrollo de las áreas urbanas, especialmente mediante acciones de mejora y conservación de sus medinas históricas. De hecho, las principales acciones de protección del patrimonio en Marruecos se concentran en las medinas de las llamadas ciudades imperiales (López Lara, 2005).

El resultado es un proceso de continuo deslavazamiento de los lazos entre las poblaciones locales y su medio natural que se deja notar en formas de abandono y deterioro rural que afectan a algunos municipios de la región. En este sentido juegan en contra el hecho de que las intervenciones tanto públicas como privadas han sido muy limitadas en el Medio Atlas Oriental, con lo que se mantiene el estatus de área remota, aislada y marginada.

Sin embargo, los recursos patrimoniales, tangibles e intangibles, que presenta el área del nordeste del Medio Atlas le da una función territorial potencial: la de emergente zona turística. El desgaste intensivo de los recursos y la exclusión del territorio de las estrategias de desarrollo socioeconómico marroquíes, salvo un número muy limitado de intervenciones públicas y privadas, han agravado los desequilibrios ecológicos, sociales y culturales mantenidos tradicionalmente en el nordeste del Medio Atlas. 
Cuestión que origina un impacto negativo en los recursos y en la cohesión social del territorio que se mantiene fuera de los procesos de desarrollo económico y social del país. Se hace cada vez más patente la dependencia de la relación del entorno regional, siendo los sistemas de producción tradicionales y los medios empleados rudimentarios. Como consecuencia, la productividad sigue siendo baja y limitada, lo que da lugar a un intensivo éxodo rural.

Los síntomas de la crisis se pueden resumir en la falta de condiciones favorables para la dinámica socio-cultural y económica para la acumulación de bienes, que se traduce en una economía pobre con bajo impulso.

Aspectos que se han detectado en las encuestas y entrevistas realizadas por Baali (2014), en las comunidades rurales de Bou Iblane, Ighzran, Ras lkssar, Berkin, Maghrawa, Assmia, Talzamt, Adrej, Dar Alhamra, Tazarin y Zrarda, cuyos resultados se resumen seguidamente:

- Una fuerte influencia antrópica en un entorno frágil y vulnerable que, como resultado, da lugar a una continua y preocupante degradación de los recursos naturales. La presión humana sobre estos espacios y paisajes forestales es alarmante $\mathrm{y}$, de hecho, el mismo patrimonio cultural se encuentra en un avanzado estado de degradación.

- La movilidad de personas y mercancías es baja, con unos flujos extremadamente débiles, en función de la pésimas infraestructuras, de la endeblez y carestía de los equipamientos básicos y de su carestía.

- Una pirámide de población joven, con baja productividad. El potencial de mano de obra está insuficientemente explotado: el $85 \%$ de los jóvenes encuestados trabajan en la agricultura y sólo un $28 \%$ pertenece o se integra en asociaciones o cooperativas locales.

- Se registran tasas de analfabetismo altas que se conjugan con unas tasas muy bajas de escolarización, especialmente de la población femenina. Con una tasa media del $51 \%$, las máximas y mínimas son, respectivamente, $60 \%$, registrada en Tafjight y $34 \%$, en Ighzrane. La tasa media de matriculación escolar es del $47 \%$, siendo las máximas y mínimas, respectivamente el $76 \%$, en Ighzrane y el $33 \%$ en Bou Iblane. La tasa media de la matriculación femenina es del $20 \%$, con un arco que va de un máximo del $36 \%$ a un mínimo del $9 \%$, registrados respectivamente en Ighzrane y Barkine.

- Las deficiencias y la falta de intervención del Estado en el desarrollo territorial refuerza la precariedad y la vulnerabilidad de las poblaciones locales, junto a la ineficiencia de las asociaciones de desarrollo.

- Se detectan formas de abandono y deterioro rural que afectan a aduares y a las comunidades más aisladas.

Por otra parte, la situación socioeconómica, según señalan los indicadores de vulnerabilidad y pobreza, es negativamente crítica. Casi todas las comunidades rurales de esta zona montañosa presentan índices de fragilidad y pobreza precarios, notablemente por encima de la media nacional (Figura 1). 
La situación es especialmente crítica en las comunidades de Oulad Ali y de Meghraoua, donde la tasa de pobreza son de un $22 \%$ y de un $24 \%$, respectivamente. En contraposición, la tasa es de aproximadamente 14,5\% en Ain Leuh, $16 \%$ en Timahdite, 22\% en Dayet Aoua y un 15\% en Tizguite, en el Medio Atlas centro-oeste (El Bezzari, 2014).

Esta región montañosa se caracteriza por la riqueza, diversidad y especificidad del patrimonio territorial, es decir, de sus recursos naturales, paisajísticos y culturales, que podría ser una poderosa palanca para el impulso del desarrollo económico y social de la región (Tribak y Otros, 2013).

\section{Figura 1}

\section{NIVELES DE VULNERABILIDAD Y POBREZA DE LAS COMUNIDADES RURALES DEL MEDIO ATLAS NORORIENTAL (2007)}

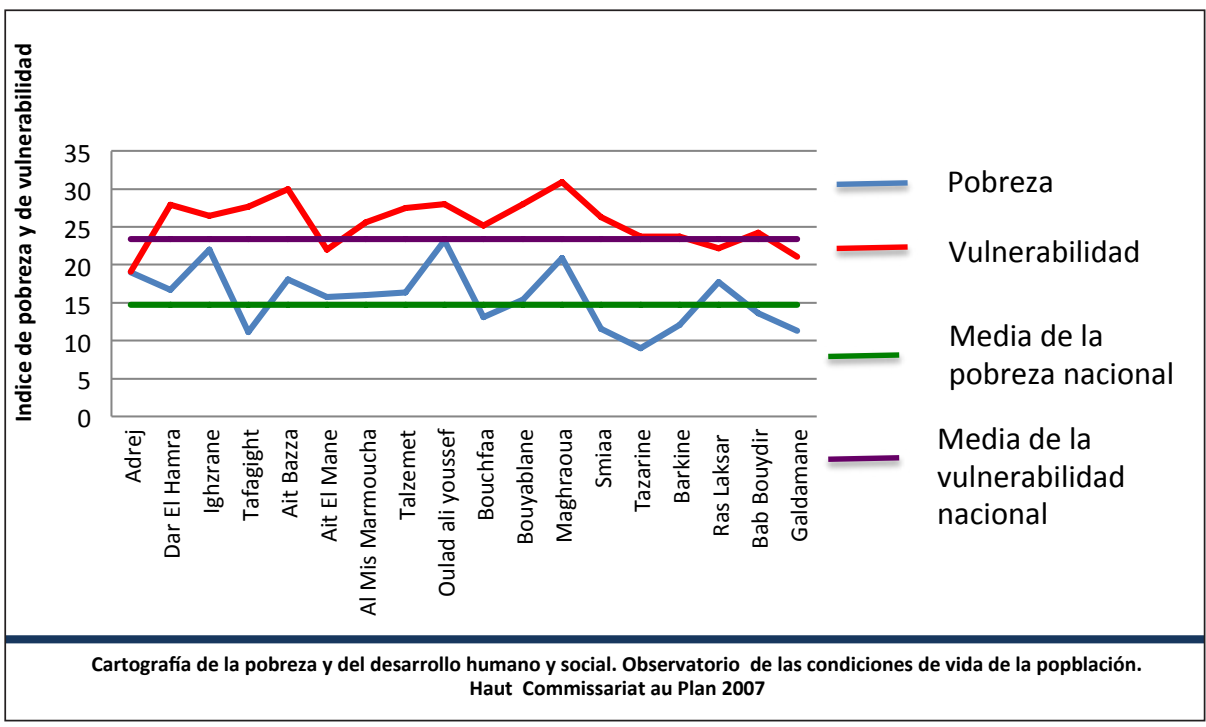

Fuente: El Bezzari, 2014.

\section{EL TURISMO: POTENCIAL ACTIVIDAD PARA EL DESARROLLO ECONÓ- MICO Y LA CONSERVACIÓN DEL PATRIMONIO TERRITORIAL}

\subsection{Análisis DAFO del turismo como recurso en la región}

Las actividades turísticas basadas en el rico patrimonio territorial y cultural representan una de las posibles actuaciones frente a la decadencia que experimentan actualmente las montañas de Marruecos (Saïgh Bousta, 2006), si bien generalizar esta opción en todas las regiones del país representa una orientación del desarrollo simplista e ingenuamente optimista (Landel y Senil, 2009).

Ha de tenerse en consideración, por un lado, la individualidad y especificad de las diferentes montañas y, por otro, las dificultades inherentes a los espacios de montañas 
para desarrollar una dinámica turística, al margen de los grandes y tradicionales destinos turísticos de Marruecos, como son las zonas costeras del Atlántico y del Mediterráneo y las grandes ciudades imperiales.

De la misma forma, adoptar esta visión para el desarrollo del nordeste del Medio Atlas como si fuera la única respuesta posible a la crisis de este territorio, sin tener en cuenta las limitaciones de contexto o externas y las internas, sería errar aún a sabiendas de la relevancia de los caracteres y de las especificidades naturales y socio-culturales locales.

Por ello, se ha de valorar si es razonable que el turismo sea la actividad fundamental que puede desencadenar el proceso de revitalización y desarrollo del nordeste del Medio Atlas, que, como derivada, lleve a consideración de si es posible utilizar el potencial de esta actividad para activar el desarrollo socio- económico y conservar los recursos ecológicos.

Recientemente, la sociedad marroquí, está experimentando un incipiente y creciente, aunque aún limitado comparado con otros países, cambios en los hábitos de ocio que responde a las crecientes tasas de urbanización, al éxodo rural, al aumento de la motorización, al incremento económico de ciertos sectores de la sociedad marroquí, entre otras causas. Estos cambios de los patrones de ocio suponen una nueva mirada sobre el territorio rural, que indican tendencias a nuevos usos del mismo a partir del ocio y del turismo (Cánoves y Otros, 2005).

Se denota una mayor necesidad de actividades en contacto con la naturaleza, en pro de su re-descubrimiento, de nuevas vivencias, aventuras y experiencias conforme a las nuevas demandas de la sociedad. Actualmente, aunque este cambio presente una tendencia al alza, si bien choca con la realidad ya que las posibilidades de contacto con la naturaleza son limitadas. Una encuesta realizada por el Ministerio de Turismo de Marruecos señala que sólo un 10,60 \% de los turistas marroquíes optan en su elección para su ocio por el turismo rural y/o de montaña.

La realización de un análisis DAFO del papel de turismo en el Medio Atlas Nororiental presenta, de manera esquemáticamente expuesta, los siguientes resultados (López Lara y Tribak, 2013):

- Fortalezas:

o riqueza y variedad del patrimonio natural y cultural,

o proximidad a Europa,

o el valor de las prácticas artesanales,

o estacionalidad poco acusada,

o marco político estable,

o clima adecuado y

o el papel que pudieran llegar a tener las asociaciones locales.

- Debilidades:

o atonía y carácter débil del mercado interior,

o escasa promoción y visibilidad exterior,

o debilidad de la infraestructura turística y

o falta de canales adecuados de distribución y promoción. 
- Oportunidades:

o potencialidades de las actividades turísticas que pueden desarrollarse,

o marco inversor favorable,

o nueva restructuración de destinos turísticos a nivel mundial,

o cooperación al desarrollo y

o políticas europeas con países vecinos, en especial, el Magreb.

- Amenazas:

o vulnerabilidad derivada de las modas,

o competidores potenciales de las regiones de montaña de Marruecos,

o profesionalización del sector,

o degradación ambiental,

o falta de transparencia en los procesos y

o amenazas latentes actualmente en la imagen de los territorios musulmanes.

Tiene sentido apostar por la actividad turística en el nordeste del Medio Atlas marroquí para incentivar y vehicular los mecanismos que, a su vez, fomente un proceso de desarrollo en la zona. Pero para ello es necesario reflexionar sobre las consecuencias que puede llegar a tener si se carece de planificación, si prevalece la falta de previsión y si el proceso no se realiza con la suficiente inteligencia y tacto ya que podría poner en peligro los propios caracteres naturales, las estructuras sociales y las tradiciones y valores culturales heredados y hoy día existentes.

\subsection{Condiciones de la integración del turismo en los procesos económicos y en la conservación del patrimonio territorial}

Todos los recursos del patrimonio territorial, natural y cultural pueden ayudar a impulsar el desarrollo de esta región económicamente empobrecida (Peyron, 2004). La puesta en valor de estos recursos es condición necesaria para la promoción del turismo.

Las actividades turísticas relacionadas con el consumo de productos tradicionales pueden crear vínculos con otros sectores económicos mediante la promoción de la comercialización y de los productos locales. Sin embargo, el desarrollo turístico de esta zona de montaña debe garantizar, por un lado, la racionalidad en la gestión del patrimonio local y su sostenibilidad y, por otro, la equidad en la distribución espacial de sus efectos socioeconómicos.

Por lo tanto, el desarrollo de actividades turísticas a pequeña escala puede ser un eficaz recurso para este entorno frágil e históricamente desheredado. Un proceso de fomento del turismo planificado basado en el compromiso de la inversión privada podría lanzar el dinamismo económico y, es probable, tenga un impacto positivo en otros sectores como la agricultura y la artesanía (Boujrouf, 2004).

Sin embargo, el turismo no puede pretender desempeñar por sí sólo un papel principal en cualquier estrategia de desarrollo. Debe ser un componente, entre otros, en el contexto de una política de desarrollo local, basada en la complementariedad, la integración y la apertura. 
El despegue del turismo en sintonía de sostenibilidad en el nordeste del Medio Atlas, debe tener un impacto positivo en la economía de la región, en función de una serie de condicionantes:

- La existencia de un umbral de densidad específica para las infraestructuras necesarias para activar las actividades turísticas; el correcto despegue de estas actividades dependerá de la adecuación, eficacia, función y ubicación de estas infraestructuras.

- La necesidad de apoyar estrategias de difusión y promoción de las actividades turísticas. El potencial turístico del noreste del Medio Atlas es prácticamente desconocido por los posibles usuarios tanto a nivel nacional como internacional. Los programas de acción para el turismo deben tener en cuenta las expectativas de los visitantes y un adecuado enfoque de marketing basado en la promoción turística de los productos específicos de la región.

- El aprovechamiento de una base local de las actividades productivas de bienes y servicios que combinan la autenticidad e innovación de interés para el turismo.

- Crear y potenciar un tejido de estructuras cohesivas y de integración social y local. Las cooperativas y asociaciones deben ser las gestoras de los proyectos de turismo en la región.

- Un marco de organización administrativa que incorpore el gobierno local en la planificación y realización de proyectos turísticos. La voluntad colectiva para participar en el mismo proyecto de desarrollo turístico es necesaria, con un proyecto que movilice la cooperación de todas las partes interesadas en la lógica de asociación, la cooperación y la flexibilidad. También es esencial que una política coherente de gestión territorial se establezca entre los diferentes niveles de toma de decisiones.

El turismo en su integración en el sistema de desarrollo local debe pasar por dos fases principales (Gráfico 2):

1. Una primera fase, correspondiente a la iniciación, donde el turismo empieza a depender de un previsión organizativa, técnica y diversificada del tejido productivo, con la presencia de cuadros intermedios técnicos y de organización. Lo que lleva a la densificación de actividades productivas y de estructuras de cohesión social.

2. Lo que daría pie a una segunda fase, la de consolidación, con el desarrollo y emergencia de actividades turísticas sostenibles y la activación de asistencia e inversión a nivel de cuadros intermedios técnicos y de organización. Llevaría a la consolidación de un tejido productivo para el desarrollo local.

Por lo tanto, el turismo y otras actividades productivas pueden apoyarse mutuamente y mantener una eficiente dinámica de desarrollo local. Esta integración de actividades turísticas podría contribuir al desarrollo de productos y otras actividades de promoción.

Por otra parte, el turismo sostenible debe basarse, principalmente, en los enfoques de intervención sectoriales más amplios que tengan en cuenta aspectos sociales y ambientales. Estos, cuando no son favorables, constituyen un factor de degradación del medio ambiente y de desintegración de las empresas locales (Clarimont y Vles, 2006). 
Asimismo es necesaria la integración económica. Para ello se requiere que las actividades turísticas se lleven a cabo en paralelo con las actividades tradicionales en la zona, como la agricultura, el pastoreo y la artesanía, integrado en un plan de desarrollo regional más amplio, con el fin de impulsar las sinergías y complementariedades entre los diferentes sectores (Labyad, 2006).

Por lo tanto, es esencial que los programas de acción y el desarrollo de proyectos de turismo sostenible se basen en las experiencias habidas en otros lugares de la montaña marroquí; un ejemplo interesante es que representa el valle de Ait Bouguemaz (Alto Atlas). De acuerdo con las entrevistas realizadas en un municipio del citado valle, Tabant, entre los turistas, los habitantes y los propietarios de casas de alquiler muestra que las actividades turística se presentan como altamente selectivas de la que se beneficia una minoría de la población local, la que posee las condiciones para establecer actividades de recepción, restauración, asesoramiento a los turistas...

\section{Gráfico 2}

MODELO DE INTEGRACIÓN DEL TURISMO EN EL DESARROLLO LOCAL

\begin{tabular}{|c|c|}
\hline FASE INICIAL & FASE DE CONSOLIDACIÓN \\
\hline $\begin{array}{c}\text { Presencia de cuadros intermedios técnicos } \\
\text { y de organización }\end{array}$ & \\
\cline { 1 - 2 } $\begin{array}{c}\text { Densificación de actividades productivas y } \\
\text { de estructuras de cohesión social }\end{array}$ & \\
\hline Despegue turístico & $\begin{array}{c}\text { Desarrollo y emergencia de actividades } \\
\text { turísticas sostenibles }\end{array}$ \\
\hline \multicolumn{2}{|c|}{$\begin{array}{c}\text { Activación de asistencia e inversión a } \\
\text { nivel de cuadros intermedios técnicos y de } \\
\text { organización }\end{array}$} \\
\cline { 2 - 2 } & $\begin{array}{c}\text { Consolidación de un tejido productivo } \\
\text { para el desarrollo local }\end{array}$ \\
\hline \multicolumn{2}{|c|}{ INTEGRACIÓN DEL TURISMO EN EL DESARROLLO LOCAL } \\
\hline
\end{tabular}

Fuente: elaboración propia.

Asimismo, el consumo de productos agrícolas por parte de los turistas extranjeros se muestra muy limitado lo que implica una articulación difícil entre la supuesta introducción del turismo y el despegue de la economía local, al menos, en la situación actualmente.

Los turistas entrevistados, que pasan por las agencias de viajes señalan que hacen la mayor parte de sus compras de productos de artesanía en las principales ciudades como Marrakech, donde los productos se diversifican y con más posibilidades de elección (El Bezzari, 2014). La población local, con sólo unas ganancias minoritarias se ve propensa a formas de alienación, lo que genera un rechazo de estas actividades por parte de las poblaciones locales que se muestran preocupadas por la desintegración de sus sociedades ya que podría resultar una pérdida de la cohesión social y sus valores ancestrales here- 
dados a largo de los siglos. Por lo tanto, es necesaria la integración social y cultural, con el apoyo de la población a través de un enfoque participativo que garantice una gestión local transparente.

La integración ecológica, no es menos importante, al requerir un turismo sostenible, en este entorno frágil y vulnerable, garante del respeto por el medio ambiente. Los paisajes naturales (paisajes forestales, sitios geológicos y geomorfológicos...) deben quedar protegidos contra cualquier forma de degradación producto de las actividades humanas y de los flujos turísticos. En este contexto, tienen que armonizarse el uso de los recursos y la necesidad de su protección.

\section{CONCLUSIONES}

Las consideraciones realizadas sobre la integración del turismo en el desarrollo local en el nordeste del Medio Atlas constatan que coexiste una situación paradójica que enfrenta un potencial basado en el patrimonio natural y cultural con una alarmante situación de precariedad que afecta tanto al medio como a la población y al capital técnico y organizativo (Gráfico 3).

\section{Gráfico 3 \\ INTEGRACIÓN DEL PATRIMONIO EN EL DESARROLLO LOCAL: SITUACIÓN COMPLEJA Y PARADÓJICA}

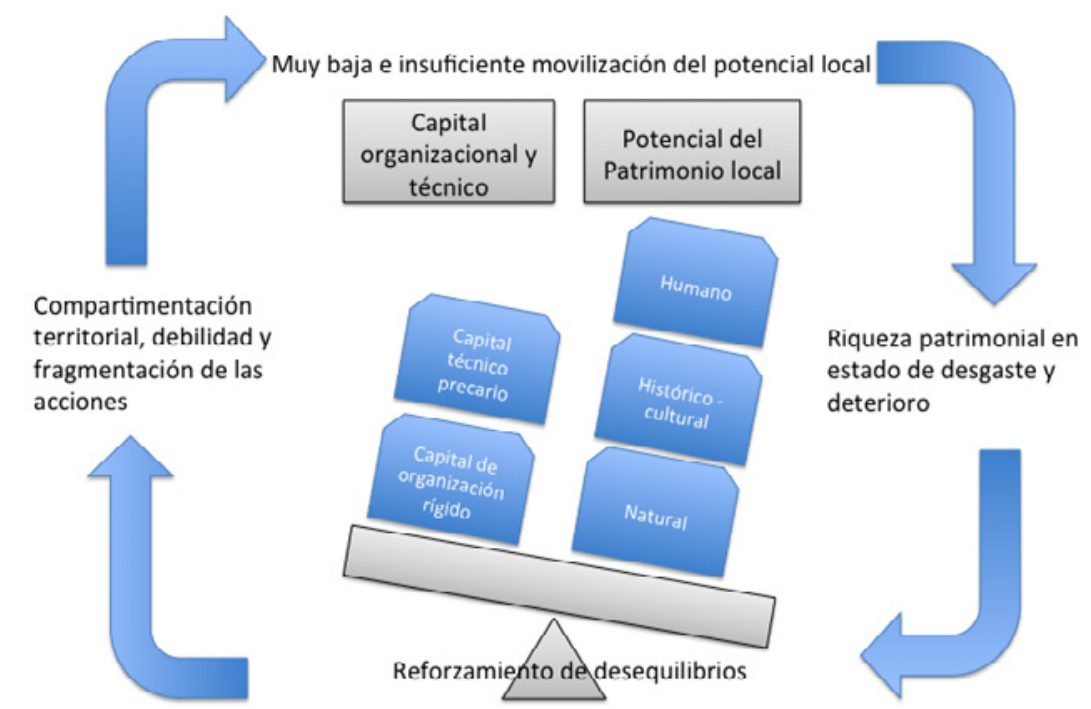

Fuente: elaboración propia.

De esta paradoja nace un desequilibrio territorial y económico que explica, en gran medida, el paulatino deterioro del patrimonio natural. Las condiciones de vida siguen estando por debajo de las expectativas de la población local, reflejando una inadecuada 
movilización del potencial local. Este estado de cosas explica la fuerte presión existente sobre los recursos territoriales locales y sobre el patrimonio natural con el corolario de la pérdida progresiva de este patrimonio, que viene a reforzar el mencionado desequilibrio. Las soluciones, de este modo, están relacionadas a cuestiones derivadas del desarrollo local.

Por lo tanto, se hace necesaria una puesta en valor turístico de los diversos recursos territoriales en búsqueda del desarrollo local, que debe basarse y estar auspiciado por distintos agentes procedentes tanto del ámbito institucional como de la iniciativa privada.

La puesta en funcionamiento de actividades turísticas deber establecer conexiones entre la población local y el resto del territorio marroquí así como con el mundo exterior. Ha de buscarse una planificación basada en la concertación y en la conciliación entre los operadores afectados por los proyectos de desarrollo turístico.

El Estado, las asociaciones locales, la población local y los inversores deben colaborar conjuntamente para garantizar el éxito de una planificación turística, con la voluntad común de liderar el desarrollo económico de la región y de obtener para las poblaciones locales empleos y fuentes suplementarias de rentas.

En este sentido, el Estado debe incentivar a los inversores locales proporcionándoles un apoyo técnico adaptado y específico a sus necesidades (información, formación, ayuda a la inversión turística...) con ayudas tendentes a la creación y adaptación de infraestructuras turísticas tales como pistas para senderismo, alojamientos rurales, etc.

La participación eficiente de sus habitantes y la responsabilidad de operadores locales representan requisitos básicos. Se debe garantizar la inserción de las poblaciones en los distintos proyectos, el desarrollo turístico no debe depender exclusivamente de inversores exógenos o funcionarios administrativos que no son la zona.

Las actividades turísticas deben tener un papel intrínseco en el desarrollo de esta región marginada del Medio Atlas oriental. Pueden contribuir al crecimiento de los niveles económicos permitiendo la integración de la región en sus contextos y favorecer vínculos culturales y territoriales entre las sociedades rurales y de montaña y el mundo exterior.

También requiere medidas para salvaguardar, conservar y popularizar el producto a una gran sector de la población. Estas acciones de conservación y mejora del desarrollo de los recursos del patrimonio deben organizarse dentro de un proyecto conjunto cuyos objetivos fueran el fortalecer la gestión del patrimonio, el desarrollo de los ingresos del turismo nacional e internacional con el fin de mejorarlos y beneficiar en primer lugar a las comunidades locales y a los actores interesados en la conservación de los valores patrimoniales y en el desarrollo local.

Por lo tanto, el establecimiento de un desarrollo turístico debe hacerse en un marco descentralizado, que pueda promover sinergia entre los diferentes actores, que están llamados a trabajar conjuntamente para asegurar el éxito de los proyectos comunes a partir de una única voluntad para desbloquear el actual estadio de estancamiento del desarrollo económico de la región y proporcionar a la población local las condiciones de trabajo que sean fuente de ingresos adicionales y necesarias para activar el desarrollo local.

De la misma forma, la opción de apostar por el turismo como una oportunidad de desarrollo en esta zona nororiental del Medio Atlas requiere precauciones para prevenir posibles impactos negativos sobre la identidad y las estructuras sociales de las comunidades locales. 


\section{BIBLIOGRAFÍA}

BAALI, H. (2014): Développement local au Moyen Atlas nord oriental: Défis, enjeux territoriaux et perspectives. Thèse de Doctorat en Géographie d'Aménagement Régional, FLSH Sais - Fès; 332 p.

BOUJROUF, S. (2004): "Tourisme de montagne au Maroc : enjeux de la durabilité", in Saïgh Bousta, R., Albertini, F. (Dir.) y Boujrouf, S. (Coord.), Le tourisme durable, réalités et perspectives marocaines et internationales. Centre de Recherches sur les Cultures Maghrébines. Université Cadi Ayyad, Marrakech, pp. 273-284.

CÁNOVES VALIENTE, G. y Otros (2005): “Turismo rural en España: paisajes y usuarios, nuevos usos y nuevas visiones". Cuadernos de Turismo, n 15 , pp. 63-76.

CLARIMONT, S. y VLES, V. (2006): "Les enjeux de la durabilité et de la gestion intégrée des territoires touristiques dans les Pyrénées Analyse comparée transfrontalière". Turismo sostenible en zonas de montaña. Université de Pau et des Pays de l'Adour. Pau, Francia, pp. 9-36.

EL BEZZARI, L. (2014): Le développement territorial au Moyen Atlas Centro-occidental : atouts, contraintes et enjeux d'acteur. Thèse de Doctorat en Géographie d'Aménagement Régional, FLSH Sais - Fès; 270 p.

FERIA TORIBIO, J.M. (2010): "Patrimonio territorial y desarrollo sostenible: un estudio comparativo en Iberoamérica y España”. Estudios Geográficos, n 268, pp. 129-159.

LABYAD, H. (2006): "Dynamique territoriale et développement local : autour des fondamentaux d'un tourisme durable dans le Haut Atlas marocain". Turismo sostenible en zonas de montaña. Université de Pau et des Pays de l'Adour. Pau, Francia, pp. 156-184.

LANDEL, P.A. y SENIL, N. (2009): Patrimoine et territoire, les nouvelles ressources du développement". Développement durable et territoires [En ligne], Dossier 1212009. URL: http://developpementdurable.revues.org/7563; DOI: 10.4000 / développement durable.7563

LÓPEZ LARA, E. (2005): "La imagen turística de Marruecos proporcionada por Internet: reinterpretando el orientalismo". Cuadernos de Turismo, nº16, pp. 123-134.

LÓPEZ LARA, E. y Otros (2007a): "Planificación y gestión del turismo ecológico en el Medio Atlas Oriental de Marruecos". Treballs de la Societat Catalana de Geografia, $n^{\circ}$ 64, pp. 51-68.

LÓPEZ LARA, E. y Otros (2007b): “Aspectos hidrológicos de la Depresión de Marhrauoa y sus márgenes (Medio Atlas Septentrional de Marruecos). Nimbus, n 19-20, pp. 124-141.

LÓPEZ LARA, E y OBDA, Kh. (2012): "Estudio de casos de hábitat rural en las montañas del medio Atlas y del Rif (Marruecos)". Anales de Geografía, vol. 32, n 1, pp. 29-43.

LÓPEZ LARA, E. y TRIBAK, A. (2013): "Establecimiento de la ruta turística TazaBouiblane como instrumento para la promoción del turismo rural y ecológico en el Medio Atlas nor-oriental (Marruecos)", Cuadernos de Turismo, no 31, pp. 175-198.

MILIAN, J. (2007): "Le dilemme entre développement et protection dans les montagnes du Maroc - le cas des parcs du Moyen Atlas”. Géocarrefour, Vol. 82/4, pp. 177-186.

PEYRON, M. (2004): "L'éco-tourisme comme levier de développement des ressources territoriales : le cas des massifs orientaux de l'Atlas marocain", Montagnes Méditerranéennes, $\mathrm{n}^{\circ} 20$, pp. 187-194. 
REINO DE MARRUECOS, (2006): Informe del Cincuentenario: 50 años de desarrollo humano y perspectivas para 2025. www.rdh50.ma; documento de síntesis en español www.rdh50.ma/esp/docsynthese.esp.pdf .

SAÏGH BOUSTA, R. (2006): "Tourisme de montagne : Quel cadre ? Quel potentiel ? Quelles perspectives ?". En Collectif tourisme durable de montagne réalités et perspectives. Publication coordonnée par Boujrouf, S. et Tebbaa, O. publication EDIT, LERMA, ERCPT et Faculté des Lettres et des Sciences Humaines, Marrakech, pp. 11-16.

SILVA PÉREZ, R. y FERNÁNDEZ SALINAS, V. (2008): "El patrimonio y el territorio como activos para el desarrollo desde la perspectiva del ocio y del turismo". Investigaciones Geográficas, nº 46, pp. 69-88.

TRIBAK, A. (2011): "Il patrimonio di prossimità del Marocco rurale : una risorsa da valorizzare per lo sviluppo locale nel mediterraneo", in La valorizzazione del patrimonio di prossimità tra fragilità e sviluppo locale, un approccio multidisciplinare. Coord. Nicoletta Buratti et Claudio Ferrari . Economia e management della cultura e creatività. Editino Franco Angeli, Milano Itali, pp. 181-204.

TRIBAK, A. y Otros (2006): “Activités touristiques et développement durable dans un espace montagnard marocain : cas du Moyen Atlas au sud de Taza (Maroc)". Turismo sostenible en zonas de montaña. Université de Pau et des Pays de l’Adour. Pau, Francia, pp. 438-448.

TRIBAK, A. y Otros (2010): Potentialités éco touristiques du Moyen Atlas Nord-oriental (Guide touristique). Publication de la Faculté des Lettres et des Sciences Humaines Saïs Fès, Sais-Fès. pp. 71.

TRIBAK, A. y Otros (2013): "Ressources patrimoniales, tourisme durable et développement local dans le Moyen Atlas Nororiental: cas de l'ensemble montagnard Bouyblan", en Ressources patrimoniales et développement local au Maroc et en Andalousie (Espagne). Publication de la Faculté des Lettres et des Sciences Humaines - Saïs Fès, $\mathrm{n}^{\circ} 26$, pp. 153-169.

TROITIÑO VINUESA, M.A. y TROITIÑO TORRALBA, L. (2016): “Patrimonio y turismo: reflexión teórico conceptual y una propuesta metodológica integradora aplicada al municipio de Carmona (Sevilla, España). Scripta Nova. Revista Electrónica de Geografía y Ciencias Sociales. (En línea). Barcelona. Universidad de Barcelona. 1 de septiembre de 2016. Vol. XX, n 543 http://www.ub.es/geocrit/sn/sn-543.pdf 